\title{
Path Planning Methods for Adaptive Sampling of Environmental and Acoustical Ocean Fields
}

\author{
Namik Kemal Yilmaz ${ }^{*}$, Constantinos Evangelinos ${ }^{\dagger}$, Nicholas M. Patrikalakis ${ }^{\dagger}$, Pierre F. J. Lermusiaux ${ }^{\ddagger}$, \\ Patrick J. Haley ${ }^{\ddagger}$, Wayne G. Leslie ${ }^{\ddagger}$, Allan R. Robinson ${ }^{\ddagger}$, Ding Wang ${ }^{\dagger}$ and Henrik Schmidt $^{\dagger}$ \\ *Department of Mechanical Engineering \\ Massachusetts Institute of Technology, Cambridge, MA 02139-4307 \\ Email: nkyilmaz@mit.edu \\ ${ }^{\dagger}$ Massachusetts Institute of Technology, Cambridge, MA 02139-4307

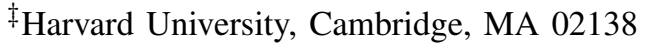

\begin{abstract}
Adaptive sampling aims to predict the types and locations of additional observations that are most useful for specific objectives, under the constraints of the available observing network. Path planning refers to the computation of the routes of the assets that are part of the adaptive component of the observing network. In this paper, we present two path planning methods based on Mixed Integer Linear Programming (MILP). The methods are illustrated with some examples based on environmental ocean fields and compared to highlight their strengths and weaknesses. The stronger method is further demonstrated on a number of examples covering multi-vehicle and multi-day path planning, based on simulations for the Monterey Bay region. The framework presented is powerful and flexible enough to accommodate changes in scenarios. To demonstrate this feature, acoustical path planning is also discussed.
\end{abstract}

\section{INTRODUCTION}

Path planning for adaptive sampling is a challenging problem and presents exciting research opportunities. There is a wide range of possible objectives for adaptive path planning in the ocean, from purely scientific to purely operational. For example, the aim can be to incorporate extra measurements that target high uncertainty areas or reduce forecast uncertainties. These extra measurements are "adaptive" since their location and nature are a function of the ocean variability and updates to the observing network. Prior works on adaptive path planning have generally different perspectives on the problem and some of them utilize optimization ideas to produce locally optimal solutions. We approach the path planning problem in an optimization framework and try to achieve a formulation and solution methodology that will generate globally optimal solutions. We test our methodology using the uncertainty data supplied by Harvard Ocean Prediction System (HOPS) [1] and Error Subspace Statistical Estimation technique (ESSE) [2][4].

In this paper we present two path planning methods for adaptive sampling based on Mixed Integer Linear Programming (MILP). The first method is predicated on network-flow ideas. An important classification of the set of path-planning problems to be solved is the number of days that are involved in the complete sampling mission. Intrinsic limitations of this first network-flow-based method prevent its use for more than a single instant. For example, computing the path for a multiple-day mission isn't directly feasible. The second method emerged by taking into consideration the limitations of the first method and is based on a general MILP formulation. It is a more powerful formulation as it: can handle multiple-day adaptive sampling missions; is scalable and can easily address the multiple vehicle case; and is more easily extended to cover unforeseen situations that might arise as adaptive sampling concepts and needs evolve. The second method also inspired techniques to be used for the solution of auxiliary problems that are required to determine suitable initial conditions for the main problem. We use the "branch and bound algorithm" which is known to be capable of providing exact solutions to MILP problems [5].

The methods are illustrated with some examples based on environmental ocean fields generated from simulations for the Monterey Bay region and AOSN-II [6] data. The framework presented is powerful and flexible and can accommodate various adaptive sampling scenarios. To demonstrate this fact, the case of an acoustical path planning is also presented.

\section{MilP Method BASEd ON Network Programming APPROACH}

The "Network Programming" approach aims at solving the optimal routing problem by combining ideas from network programming and mixed integer programming (MIP) techniques. The problem at hand, in its simplest form, is to find an optimal path for a vehicle, given its starting point and an uncertainty field coming from an ESSE solver. The termination point of the vehicle can be specified either as an exact point in space or a set of points. The method seeks a globally optimal solution. The problem can be extended to a multi-vehicle case also including inter-vehicle coordination.

The uncertainty field is a $2 D$ array, input to the problem. To implement the suggested approach, the nodes in the $2 D$ field are woven into a grid structure by introducing horizontal and vertical flows in between adjacent nodes. The introduced horizontal flows include flows from left to right (forward horizontal flows) as well as right to left flows (reverse horizontal flows). The vertical flows include top to bottom flows (forward vertical) and bottom to top flows (reverse vertical). This forms the flow grid for the network approach. 
In a network programming scheme, the node-arc incidence matrix (A) represents the connection between nodes in a network. It lays a structure for the rest of the formulation. In our particular example, each element of the uncertainty matrix is a node. The arc-incidence matrix determines which links are established between the nodes in the system. In the general case, it is a ( $\sharp$ of nodes) by ( $\sharp$ of edges) matrix. Each row corresponds to a node and each column corresponds to an arc. The $(i, k)$ th entry $a_{i k}$ is associated with the ith node and $k t h$ arc. We define $a_{i k}$ as:

$$
a_{i k}= \begin{cases}1 & \text { if } i \text { is the start node of the } k \text { th arc } \\ -1 & \text { if } i \text { is the end node of the } k \text { th arc } \\ 0 & \text { otherwise }\end{cases}
$$

Flow variables are the unknown in the problem. Therefore the solver aims to find values of these variables. Each column of A matrix corresponds to a flow variable. Let us denote by a vector $\mathbf{f}$ a particular ordering of flow variables( the columns of matrix A must follow the same ordering in order to match the flows). Then $\mathbf{A}$ and $\mathbf{f}$ are used to write down the continuity equation. To illustrate, let us focus on the ith row of $\mathbf{A}$, denoted by $\mathbf{a}_{i}$. This row is associated with node $i$. The nonzero entries stand for the arcs that are incident to node $i$. If it is an incoming arc the entry is -1 , if it is an outgoing arc then the nonzero entry is +1 . Thus,

$$
\mathbf{a}_{i}^{\prime} \mathbf{f}=\sum_{j \in O(i)} f_{i j}-\sum_{i \in I(i)} f_{j i}
$$

where $O(i)$ and $I(i)$ stand for the set of outgoing and incoming flows from and to the node $i$ respectively.

The flow conservation equation at node $i$ can be written as

$$
\mathbf{a}_{i}^{\prime} \mathbf{f}=b_{i}
$$

or, equally in matrix notation,

$$
\mathbf{A f}=\mathbf{b}
$$

where $\mathbf{b}$ is the vector $\left(b_{1},, \ldots, b_{n}\right)$ and $b_{i}$ is the amount of flow that enters the network at node $i$. In general, node $i$ is called a source if $b_{i}>0$ and a sink if $b_{i}<0$.

In our formulation all $b_{i} s$ except at two special nodes will be zero. There will be a source node at the start location of the vehicle and a sink node at an unknown location. The location of the sink node will be determined as a part of the solution.

In order to formulate the problem at hand, it is necessary to include a virtual start node and a virtual termination node. The nodes will serve a special purpose in the formulation and thus are critical. The problem will be treated as a variation of the "all to one" shortest path problem [7]. "One" node is the starting node ('s'). The termination node is undetermined in the beginning. All the nodes in the network (or depending on the problem a subset of them) are potential termination nodes.

The starting node is connected only to the node where the vehicle starts its motion, whereas termination node is connected to all nodes. This is pictured in Fig. 1(a). To give the big picture of the solution approach, a flow of magnitude +1 will be fed into the network at the start node. Then it will follow a path that will be determined by the objective function and constraints, and will leave the network at the termination node.

Since the starting node $(s)$ is connected only once, it can be omitted by setting the value of its entry in $\mathbf{b}_{i}$ to +1 . Edges from all network nodes to $t$ are one directional and only one of the flows will be +1 through these with all others remaining equal to 0 . The non-zero flow will point to the termination node.

The cost vector is constructed using the uncertainty data. For the connection between the nodes, the cost value (which corresponds to the award that will be collected if that connection is a part of the solution) must be defined. The uncertainty is provided in a matrix denoted as $\mathbf{P}$. The elements of this matrix correspond to the uncertainty value at the corresponding grid location. To extract the cost associated with a flow, the average of the uncertainty values at the start and end nodes are taken.

$$
c_{i-j}=c_{j-i}=\frac{P_{i}+P_{j}}{2}
$$

Each vehicle has a specific range and can only travel up to that range. To prevent a solution from violating the range restriction, an appropriate constraint must be introduced. This constraint can be written as follows:

$$
\sum f_{i} \cdot L_{i} \leq R
$$

$\mathbf{L}$ vector stands for the modifications on flow lengths in case of the inclusion of diagonal moves in the formulation and also for the case of curvilinear grid geometry. In the specific case where only horizontal and vertical moves are considered, $\mathbf{L}$ is a vector of ones. In the matrix notation the above inequality can also be expressed as

$$
\mathbf{L}^{T} \cdot \mathbf{f} \leq R
$$

Since $c_{k l}$ and $c_{l k}$ have the same value, naturally the optimal solution will tend to include both of the corresponding flows, $f_{k l}$ and $f_{l k}$. This will cause a back and forth motion along the same line. As it will be mentioned later there are integrality constraints on $f_{k l}$ and it may be either 0 or 1 . To prevent the oscillating notion, we impose the constraint $f_{i j}+f_{j i} \leq 1$. Hence $f_{i j}=1 \Rightarrow f_{j i}=0$ or vice versa.

Also we need to impose the constraint that each node can be visited only once. If this restriction is not imposed then, moves resembling the shape of number 8 (or papillon shape) become very common in the solutions found. The problem with these kinds of path geometry is the double counting of some observation point and focusing around the same area more than necessary. This can be achieved by introducing the following mathematical inequality:

$$
\sum f_{i j}+f_{j i} \leq\left(2-b_{i}\right), \quad \forall i \in N
$$




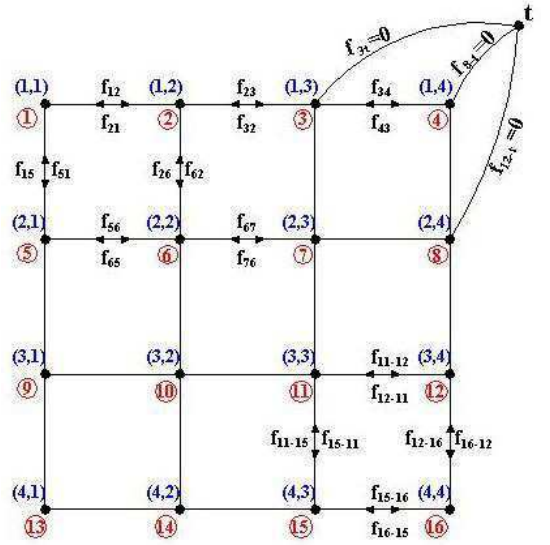

(a)

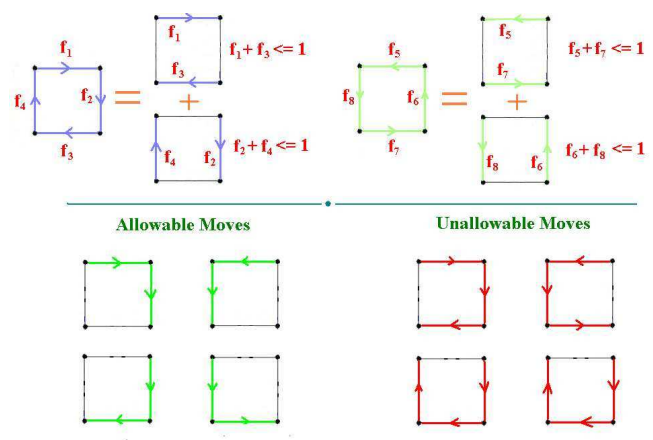

(b)

Fig. 1. a) Indexing notation for nodes and flows. b) Constraints to eliminate loops of size $1 \times 1$.

If $b_{i}$ is 0 for a node then right-hand-side of the equation becomes 2 and it means that if the node is not a source or sink node, there can be one flow entering and one flow exiting.

The subtour problem emerges in optimization problems where a route or routes need to be generated. Examples of these problems include the Traveling Salesman Problem (TSP) and the Vehicle Routing Problem(VRP) [8], [9]. In our case, similar issue arises and our remedy is to introduce special constraints that prevent the inclusion of loops in the solution. Our subtour elimination approach is based on the idea of imposing some constraints on the flows around the square loops of various sizes. The idea is illustrated on a " $1 \times 1$ loop" in Fig. 1(b).

The idea can be extended to loops of higher size as a function of the vehicle range. The complete formulation for the multi vehicle case can be written as follows:

\section{$R:$ Range}

$N$ : Network of all nodes in the grid and the start and the termination nodes.

$V:$ Set of all vehicles in the fleet

$c_{p, i-j}=\frac{1}{2}\left(P_{p, i}+P_{p, j}\right):$ Cost of moving from node $i$ to node $j$ for vehicle $p$.

$\boldsymbol{A}$ : Connectivity matrix.

$f_{p, i-j}$ : Flow from node $i$ to node $j$ belonging to vehicle $p$. $s:$ Starting node for network flow problem.

$t$ : Termination node for the network flow problem.

$$
\max \sum c_{p, i-j} f_{p, i-j}
$$

subject to

$\sum f_{p, i-j}-\sum f_{p, j-i}=b_{p, i} \quad \forall(i-j) \in N \quad \forall p \in V$

or in an equivalent representation $\mathbf{A f}_{p}=\mathbf{b}_{p}$ where $b_{p, s}=$ $\left.1 \quad b_{p, t}=-1 \quad b_{p, i}=0 \quad i \neq s, t\right)$

$$
\sum f_{p, i-j} \cdot L_{p, i-j} \leq R
$$

$\forall(i-j)(k-l) \in N$ and $\quad \forall p \in V$ where $L_{i-j}$ is a distance modification matrix to take care of diagonal moves if they are allowed and the curvilinear grid geometry in case it is used.

To disallow opposite flows between two nodes, we use the constraint:

$f_{p, i-j}+f_{p, j-i} \leq 1 \quad \forall(i-j) \quad$ and $\quad(j-i) \in N \quad \forall p \in V$

To avoid visiting the same node twice, we use the constraint:

$$
\begin{aligned}
\sum_{j} f_{p, i-j}+f_{p, j-i} \leq\left(2-b_{i}\right) \quad \forall i \in N, \quad \forall(i-j) \quad \text { and } \\
(j-i) \in N, \quad \forall p \in V(11)
\end{aligned}
$$

To avoid size " $\ell$ " loops, we use the constraint:

$\sum f_{p, i-j} \leq \Omega_{\ell} \quad \forall f_{p, i-j} \in S L_{\ell k}, \quad \forall S L_{\ell k} \in N, \quad \forall p \in V$

where $S L_{\ell k}$ stands for the $k^{t h}$ member of the set of all square shaped regions of size $\ell . \Omega_{\ell}$ stands for the upper limit value on the summation on circumferential flows for "size $\ell$ loops". Typical values can be exemplified as $\Omega_{1}=1, \Omega_{2}=4$ and $\Omega_{3}=6$.

To mask out of range flows, we use the below constraint where $R$ stands for nodes within the range of vehicle:

$$
f_{p, i-j}=0 \quad \forall(i, j) \in(\mathrm{N}-\mathrm{R}), \quad, \quad \forall p \in V
$$

For vicinity constraints, we add the following constraint:

$$
\sum f_{p, i-j} \leq 12\left(1-f_{q, k-l}\right)
$$

$\forall f_{p, i-j}, \quad f_{q, i-j} \in V C R_{z}, \quad \forall p, q \mid p>q \in V, \quad \forall V C R_{z} \in N$

We also need the impose the integrity constraint on the flows: 


$$
f_{p, i-j}=\{0,1\}, \quad f_{p, i-j} \text { must be integer. }
$$

The mathematical program has been implemented using the "XPress-MP" from "Dash Optimization" [10]. Fig. 2(a) presents the solution for a two-vehicle problem. The total solution takes around $60-70$ seconds on a Pentium 4, 2.8 $\mathrm{GHz} \mathrm{PC}$ with $1 \mathrm{~Gb}$ memory.

\section{General MilP Method for Multi-day Missions}

An important class of problems include multi-day missions where the optimization needs to be performed over a time window. The nature of the network programming based formulation requires the starting positions on each day to be available in the beginning, so that corresponding elements in the related matrices can be set accordingly. Unfortunately for a multi-day mission although the starting points of vehicles for first days are available, that of followings days are not and must be calculated as a part of the solution process. Therefore considering the shortcomings of the method, we developed another MILP based method. The second method is not based on the idea of weaving the physical space with flows and trying to highlight the flows that will be a part of the solution. It is predicated on the idea of segmenting the path with a number of path-points and trying to solve for the coordinates of those points. This idea is illustrated in Fig. 2(b). In this paper, we will explain the important aspects of this general formulation without going into details. The readers can refer to [11], [12] for the complete mathematical description of the methodology.

The number of path-points is a parameter that is defined as a function of vehicle range and grid dimensions. The objective function of the optimization problem is to maximize the path integral of the uncertainty values along the vehicle path. This is a rather complicated function to express given the restrictions on the kind of functions you can feed into a MILP solver. The first step in representing such a function is to define the nonconvex and non-concave uncertainty field using piecewiselinear functions. This is possible by using the concept of special ordered set of type 1 and 2 (SOS1 and SOS2) [13]. This concept is first introduced by Beale and Tomlin [14]. The objective function which is the path integral of the uncertainty values along the vehicle paths, can be written in mathematical terms as:

$$
\max \sum_{i=1}^{N} U(x(i), y(i))
$$

where $U(x(i), y(i))$ stands for the uncertainty field. After we have a valid representation for the uncertainty field, the objective function can be easily be expressed in MILP formulation [11], [12].

The next task is to write down the constraints that govern the motion of the vehicles. The first set of constraints are related to the primary motion of the vehicle by which we mean the motion the vehicle needs to make in order to move to one of the surrounding 8 nodes around it. This idea is represented in Fig. 2(c). These constraints define the relation between the coordinates of a path-point and the path-point preceding it.

There also exist constraints designed to prevent curling. These constraints define relation between the coordinates of a path-point and the path-point preceding it by two and three (the number might increase as a function of the vehicle range). This idea is represented in Fig. 3(a). A generic form of these constraints can be written as:

$$
\begin{aligned}
& \forall p \in[1, \ldots, P] \text { and } \forall i \in\left[n+1, \ldots, N_{p}\right]: \\
& \quad\left|x_{p i}-x_{p(i-n)}\right| \geq \Delta_{n} \text { OR }\left|y_{p i}-y_{p(i-n)}\right| \geq \Delta_{n}
\end{aligned}
$$

where $P$ is the total number of vehicles in the fleet and $N_{p}$ is the total number of path-points belonging to $p^{t h}$ vehicle. $n$ stands for the distance between path-points the anti-curling constraint is set for and $\Delta_{n}$ is the corresponding separation distance. In all of the above equations, subscripts $p$ and $k$ stand to denote the $k^{t h}$ path-point of $p^{t h}$ vehicle. Using formulations techniques it is possible to write the above formulation in a MILP notation [11], [12].

Vicinity requirements and collision avoidance for multivehicle scenarios can be handled with the following constraints:

$$
\begin{aligned}
& \forall p, q \in[1, \ldots, P]: \forall p, q \mid p>q ; \quad \forall i, j \in\left[1, \ldots, N_{p}\right]: \\
& \left|x_{p i}-x_{q j}\right| \geq \Delta x_{\text {safety }} \quad \text { OR }\left|y_{p i}-y_{q j}\right| \geq \Delta y_{\text {safety }}
\end{aligned}
$$

where the safety distances in $x$ and $y$ directions are denoted by $\Delta x_{\text {safety }}$ and $\Delta y_{\text {safety }}$ respectively.

There also exist constraints which are required for the coordination issues that are related to the different communication considerations with overshadowing ships, shore stations and buoys. Communication with AUVs is established by over radio signals (most commonly), acoustically, or via direct connection to the AUV by a cable. The suggested MILP formulation is strong and flexible enough to incorporate necessary constraints for these different scenarios easily [11], [12]. The need for communication with multiple platforms arises in the context of an Autonomous Ocean Sampling Network $(A O S N)$ [16], [6], [17]. AOSN is a concept that is still under development. The ultimate goal of AOSN is the realization of a completely autonomous network in charge of collecting data from the ocean. The network may consist of AUVs, buoys, shore stations, acoustic modems, satellite and radio links and other autonomous vehicles. In a simplified example scenario, the shore station makes the mission plan and sends it to the buoys via a radio link. Buoys establish an acoustical communication link with AUVS and upload the individual path plans to the AUVs. The AUVs navigate in accordance to the uploaded plan and make necessary measurements. When the mission is completed, the collected data is transmitted to one of the buoys. The buoy sends the data to the shore station using the wireless connection. Also, buoys not only act as an 


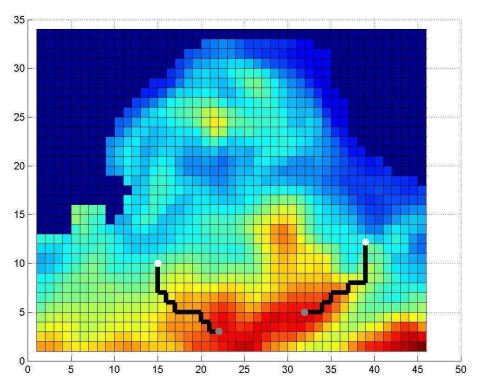

(a)

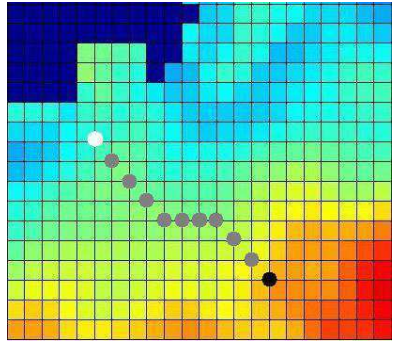

(b)

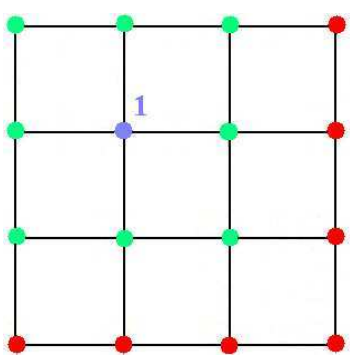

(c)

Fig. 2. a) Result for two-vehicle case. Starting coordinates and range (in grid units) for vehicle $1: x=15, y=10$, Range $=14$. Starting coordinates range (in grid units) for vehicle $2 x=39, y=12$, Range $=14$; Total Reward $=1078^{\circ} \mathrm{C}$. b) Path construction by segmentation. c) Allowable set of path-points by taking into account the spatial constraints between the candidate point and the point that precedes it by one. Blue dots represent current path-points in the path, green ones show the allowable path-point locations and the red ones show the unallowable path-point locations for the path-points under consideration.
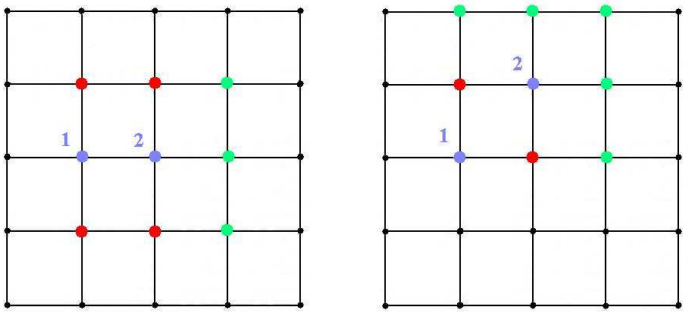

(a)

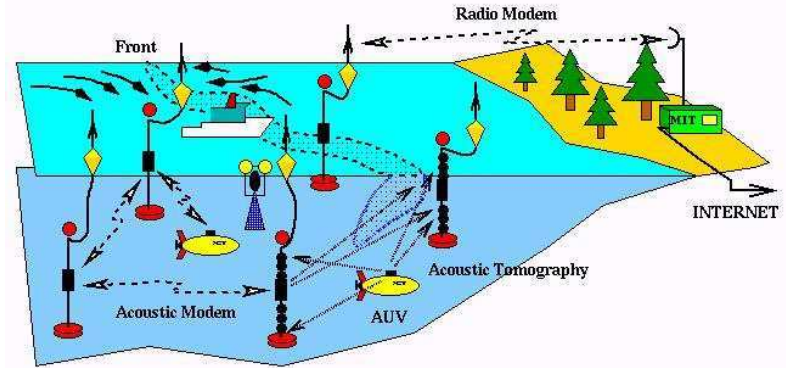

(b)

Fig. 3. a) Allowable set of path-points by taking into account the spatial anti-curling constraints between the candidate point and the point that precedes it by two. b) Illustration of an "Autonomous Oceanographic Sampling Network" [15].

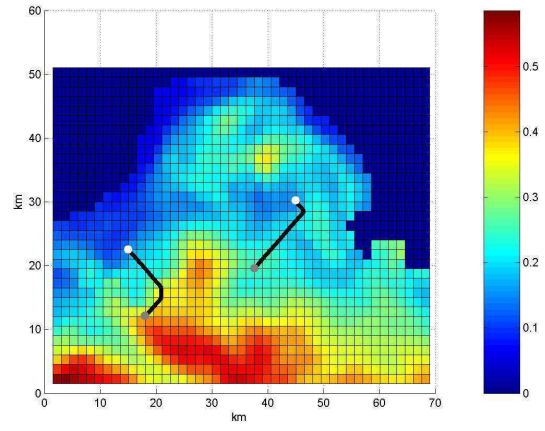

(a)

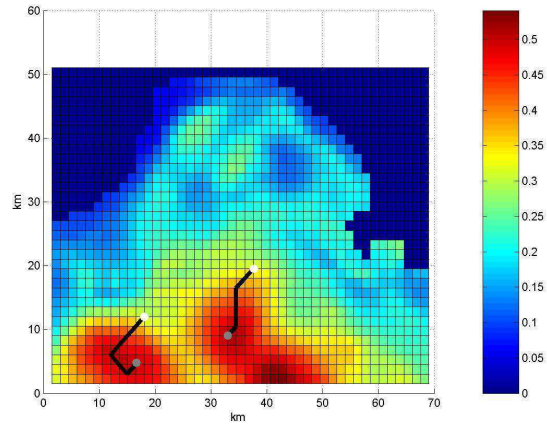

(b)

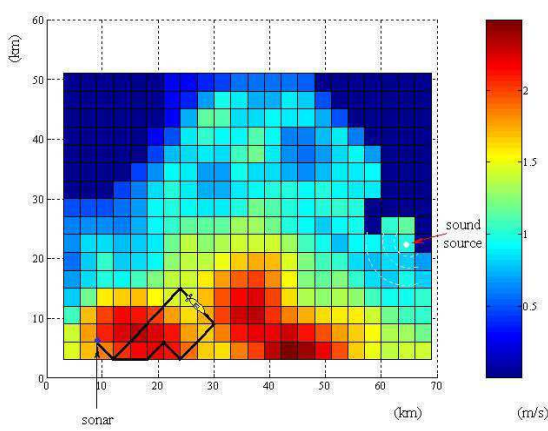

(c)

Fig. 4. a) Results for first day of a time-progressive case example. Starting coordinates: $x_{1}=15 \mathrm{~km}, y_{1}=22.5 \mathrm{~km}, R_{a n g e}=14.5 \mathrm{~km} ; x_{2}=45 \mathrm{~km}$, $y_{2}=30 \mathrm{~km}$, Range $_{2}=15 \mathrm{~km}$. b) Results for second day of a time-progressive case example. c) An acoustic problem example.

intermediate data logger but also can serve as docking stations where AUVs can be recharged and continue their mission without being needed return to the shore or board to a ship. Fig. 3(b) illustrates an AOSN.

We present a formulation in [11], [12] designed around the extended functionality of buoys as docking stations in addition to being a node in the communication network. Other scenarios and different requirements can be formulated and relatively easily implemented. This is because of the power of MILP techniques and of the flexibility of our formulation framework.

An important set of problems are the planning of missions over multiple days with a need to establish the information link between consecutive days. The general MILP formulation can easily account for this by adding a time dimension to cover multi-day problems. We use the "Mosel" modeling language from "Dash Optimization" [10] to implement the MILP problem and a new time dimension translates into adding an index to the related matrices. A very key point 
in establishing the link between consecutive days, and to introduce the time-progressive features, is to define the relation between the end path-point of vehicles on one day with the starting point on the following day. One option is to impose the constraint that on consecutive mission days the vehicles should start their mission exactly at the location they finished their mission on the previous day. This can be achieved by the following constraints:

$$
\begin{array}{r}
\forall p \in[1, \ldots, P], \quad \forall d \in[2, \ldots, D]: \\
x_{p d 1}=x_{p(d-1) N_{p}} \\
y_{p d 1}=y_{p(d-1) N_{p}}
\end{array}
$$

where $D$ is the total number of days in the mission. Fig. 4(a) and 4(b) present results for a two-day time-progressive case. This example also reveals the power of the proposed formulation to find time global optimal solutions. If we assume the absence of any information link between the uncertainty data for day 1 and day 2, on day 1 the second vehicle, which starts its motion at $\mathrm{x}=45 \mathrm{~km}$ and $\mathrm{y}=30 \mathrm{~km}$, needs to be drawn to the small peak located around $\mathrm{x}=40 \mathrm{~km}, \mathrm{y}=35 \mathrm{~km}$ causing the high-uncertainty region on day 2 to stay out of the reach of vehicle 2. But since there is communication between day 1 and day 2, vehicle 2 compromises on the total amount of reward it can collect on day 1 , and heads towards the highuncertainty region that will appear on day 2 to maximize the total reward over two days.

The MILP path planning algorithm can also be applied on an acoustical system [18] (with some modification if necessary). Fig. 4(c) shows a sound velocity prediction error map. Suppose a sonar system is at the blue point and a sound source is at the white point. From the error map, large uncertainties exist around the sonar location. The sonar may localize the sound source in a wrong bearing. It is desirable to send an AUV carrying conductivity, temperature and depth (CTD) sensors to do in-situ measurements in the region, so that after these data are assimilated, the sound velocity error is reduced and the sonar bearing localization can be expected to be more accurate. Due to AUV's performance limits, an optimized sampling path is needed to capture critical uncertainties and improve sonar performance as much as possible.

\section{CONCLUSIONS}

We developed two MILP-based methods for path-planning for adaptive sampling. Due to intrinsic shortcomings the first method was incapable of handling multi-day missions. A second general method was therefore developed. This second method can treat a larger range of problems and produce solutions faster than the first one in the case of single day missions. Also its implementation is very flexible in the sense that any modifications to the problem scenario can easily be accommodated. The framework could be utilized as a foundation for future research where we can embed approximations of HOPS and ESSE into the optimization framework to establish the link between the measurements performed at a generated path-point and their effect on uncertainty field(s) involved in the problem. This would allow the reduction of uncertainty fields dynamically: the objective (error) field is modified after each path point. The path planning framework presented in this paper could also be extended to low level path planning where vehicle dynamics can also be taken into consideration [19].

\section{ACKNOWLEDGMENT}

This work was funded in part by the National Science Foundation (under ITR grant EIA-0121263) and the Department of Commerce (under NOAA - MIT Sea Grant College Program grant NA86RG0074). PFJL, PJH and WGL thank their AOSN-II colleagues and were supported by the Office of Naval Research under grant N00014-05-1-0335, N00014-041-0534, N00014-05-G-0106 and N00014-05-1-0370.

\section{REFERENCES}

[1] "Harvard Ocean Prediction System (HOPS)," http://oceans.deas.harvard.edu/HOPS/HOPS.html.

[2] A. R. Robinson and S. M. Glenn, "Adaptive sampling for ocean forecasting," Naval Research Reviews, vol. 51, no. 2, pp. 28-38, 1999.

[3] P. F. J. Lermusiaux, "Estimation and study of mesoscale variability in the strait of sicily," Dynamics of Atmospheres and Oceans, vol. 29, pp. $255-303,1999$

[4] _ , "Evolving the subspace of the three-dimensional multiscale ocean variability: Massachusetts bay," J. Marine Systems, Special issue on "Three-dimensional ocean circulation: Lagrangian measurements and diagnostic analyses", vol. 29, no. 1-4, pp. 385-422, 2001.

[5] D. Bertsimas and J. N. Tsitsiklis, Introduction to Linear Optimization. Athena Scientific, 1997, ch. 11, pp. 485-490.

[6] “AOSN II / Monterey Bay 2003," http://people.deas.harvard. edu/ leslie/AOSNII/.

[7] D. Bertsimas and J. N. Tsitsiklis, Introduction to Linear Optimization. Athena Scientific, 1997, ch. 7, p. 332.

[8] E. L. Lawler, J. K. Lenstra, A. H. G. R. Kan, and D. B. Shmoys, The Traveling Salesman Problem, ser. Wiley-Interscience Series in Discrete Mathematics and Optimization. New York: John Wiley \& Sons, 1985.

[9] "Vehicle Routing Problem." http://neo.lcc.uma.es/radiaeb/WebVRP/.

[10] "Dash Optimization," http://www.dashoptimization.com.

[11] N. K. Yilmaz, "Path planning of autonomous underwater vehicles (AUVs) for adaptive sampling," Ph.D. dissertation, Massachusetts Institute of Technology, September 2005.

[12] N. K. Yilmaz, C. Evangelinos, P. F. J. Lermusiaux, and N. M. Patrikalakis, "Mixed integer linear programming (milp) path planning of autonomous underwater vehicles (auvs) for adaptive sampling," 2006, submitted to the IEEE Journal of Oceanic Engineering.

[13] H. P. Williams, Model Building in Mathematical Programming, 4th ed. John Wiley \& Sons, Ltd, 1999.

[14] E. M. L. Beale and J. A. Tomlin, "Special facilities in a general mathematical programming system for non-convex problems using ordered sets of variables," in Proceedings of the 5th International Conference on Operations Research, J. Lawrence, Ed., Tavistock, London, 1969.

[15] "Adaptive rapid environmental assessment (AREA): MIT, 2006," http://acoustics.mit.edu/faculty/henrik/uw_rto.html.

[16] T. B. Curtin, J. G. Bellingham, J. Catipovic, and D. Webb, "Autonomous oceanographic sampling networks," Oceanography, vol. 6, no. 3, pp. 8694, 1993.

[17] "Autonomous Ocean Sampling Network," http://www.mbari.org/aosn/default.htm.

[18] H. Schmidt, "Area: Adaptive rapid environmental assessment," in Impact of Littoral Environmental Variability on Acoustic Predictions and Sonar Performance, N. G. Pace and F. B. Jensen, Eds. Kluwer Academic Publishers, 2002, pp. 587-594.

[19] E. Fiorelli, N. E. Leonard, P. Bhatta, D. Paley, R. Bachmayer, and D. M. Fratantoni, "Multi-AUV control and adaptive sampling in monterey bay," June 2004. 